Emerging Adulthood: Developmental Stage, Theory, or Nonsense?

\author{
Moin Syed \\ University of Minnesota
}

In: J. J. Arnett (Ed.) (2015). The Oxford handbook of emerging adulthood (pp. 11-25). New York: Oxford University Press.

\begin{abstract}
Author Note
The ideas expressed in this chapter benefitted from conversations with Jeff Arnett, Malin Broberg, Andy Collins, Catherine Cooper, Ann Frisén, Megan Gunnar, Philip Hwang, Michael Maratsos, Lauren Mitchell, Tom Spencer, and Maria Wängqvist. Responsibility for the ideas in the chapter, as well as errors of omission or commission, rests with me alone. Address correspondence to Moin Syed at moin@umn.edu.
\end{abstract}




\begin{abstract}
Arnett's (2000) theory of emerging adulthood has been both widely celebrated and strongly criticized. However, it has not yet been closely scrutinized for what it claims to be: " $a$ new theory of development for the late teens though the twenties." (p. 469). The purpose of this chapter is to take up this scrutiny, evaluating some of the major postulations and criticisms of emerging adulthood in light of the available evidence. In particular, the chapter focuses on three broad claims pertaining to emerging adulthood: 1 ) that it is a developmental stage, 2) that it is a theory, and 3) that it is nonsense. The analysis presented in the chapter is not meant to resolve the debates in the field, but rather to examine the nuance and celebrate the complexity of the questions in order to stimulate further theory and research on the topic.
\end{abstract}

Keywords: Emerging adulthood, Theory, Developmental Stages 


\section{Emerging Adulthood: Developmental Stage, Theory, or Nonsense?}

"I propose a new theory of development from the late teens through the twenties, with a focus on ages 18-25. I argue that this period, emerging adulthood, is neither adolescence nor young adulthood but is theoretically and empirically distinct from them both.”

(Arnett, 2000, p. 469)

In proposing the concept of emerging adulthood, Arnett (2000, 2004) made two moves that are rarely seen in contemporary developmental science: 1 ) He proposed a new phase of the lifespan, and 2) he proposed a new grand theory through which to understand this new phase. Not surprisingly, these moves have generated not only much interest among researchers (and the public), but also much derision. Despite the fact that the theory of emerging adulthood has been both widely celebrated and strongly criticized, it has not yet been closely scrutinized for what it claims to be: "a new theory of development for the late teens though the twenties." The purpose of this chapter is to take up this scrutiny, evaluating some of the major postulations and criticisms of emerging adulthood in light of the available evidence. In particular, I evaluate three broad claims pertaining to emerging adulthood: 1 ) that it is a developmental stage, 2) that it is a theory, and 3) that it is nonsense.

\section{Emerging Adulthood Defined}

As captured succinctly by the quote at the opening of this chapter, emerging adulthood is meant to describe a new life stage for the period between adolescence and adulthood.

Importantly, it is not considered a universal life stage, but rather one that has emerged in certain industrialized societies due to social and economic changes that have led to delays in marriage, parenthood, and the assumption of other adult roles (Arnett, 2000, 2011). These changes are farranging, including increased participation in higher education, changes in attitudes towards premarital sex, increased women's rights, and profound changes in the meaning of adulthood itself (Arnett, 2004; see also Waters, Carr, Kefalas, \& Holdaway, 2011). Emerging adulthood has five defining features, according to Arnett (2004): identity exploration, in which young people are searching to find meaning in work, relationships, and ideologies; instability, which refers to individuals' tendencies to change residences, jobs, and relationships more frequently than at other times of life; possibilities captures the optimistic spirit of emerging adulthood, referring to the many options that emerging adults see before them; self-focus refers to emerging adults' relative freedom from obligations to parents, spouses and children, allowing them to pay greater attention to their own lives; and feeling in-between is indicative of the subjective experience of emerging adults who acknowledge feeling not quite like adolescents any more, but not fully like adults yet.

Although Arnett (1994, 1997, 1998; Arnett \& Taber, 1994) had been publishing about the changing nature of both adolescence and adulthood for some years, it was not until his 2000 article in American Psychologist that the theory of emerging adulthood was released to the scholarly public. The ideas he put forth were quickly celebrated. Within a few years came the first Conference on Emerging Adulthood, an authored book (Arnett, 2004), an edited book (Arnett \& Tanner, 2006), coverage in the popular media (e.g., Time and New York Times Magazine), a professional society (Society for the Study of Emerging Adulthood), a new journal 
(Emerging Adulthood), and now this Handbook. Clearly, emerging adulthood struck a chord within academia and the public at large.

The criticism of emerging adulthood, however, came just as quickly as the celebration. Indeed, debate has been endemic to the theory of emerging adulthood. At the Conferences of Emerging Adulthood there have been plenary sessions that featured debates about whether the implications of emerging adulthood are positive or negative for development, and even whether the concept is useful at all. The latter debate was published as a back-and-forth in Child Development Perspectives (Arnett, 2007a, 2007b; Hendry \& Kloep, 2007a, 2007b), and then further developed into a co-authored book (Arnett, Kloep, Hendry, \& Tanner, 2011). The first issue of the new journal Emerging Adulthood began with an exchange between Arnett (2013) and Twenge (2013) about whether emerging adults are more narcissistic and self-absorbed then ever before. In short, the theory of emerging adulthood has its fair share of critics.

In the following section, I provide some more detail about the primary criticisms of emerging adulthood. Collectively I refer to these criticisms as "the nonsense," not to disparage them, but rather to highlight how the criticisms all suggest that emerging adulthood, either in part or totality, is nonsense. Indeed, all of the points made are well-reasoned and valid, and will require serious attention from scholars as work on emerging adulthood continues.

Before I move on, however, it is important to note that I am not exactly an impartial observer. I have been involved with what would ultimately become the Society for the Study of Emerging Adulthood since 2007. I am now a member of the Governing Board of the Society and an Associate Editor of Emerging Adulthood, the Society's journal. While it is not my aim to provide a biased evaluation that favors emerging adulthood, these involvements undoubtedly color the analysis that is to follow.

\section{Emerging Adulthood as Nonsense}

Many critics of emerging adulthood seem to be operating within Sagan’s (1980) dictum, “extraordinary claims require extraordinary evidence," feeling that Arnett and others have not provided sufficient evidence to support such lofty claims of a new phase of the lifespan. The criticisms are wide-ranging, but can be organized into six broad points:

1. The ideas behind emerging adulthood are not new. This is a particularly frequent criticism among sociologists, who for some time had been charting the changing nature of the transition to adulthood (Côté, 2000; Hartmann \& Swartz, 2007; Waters et al., 2011). Indeed, in the prologue to his classic work, Identity: Youth and Crisis, Erikson (1968) seemed to foresee not only the changing nature of adulthood, but that that the change may produce a new phase of the lifespan:

...young adulthood will be divided into older and younger young adults, the not too young and not too old specialists probably moving into the position of principal arbiters - each for the limited period of ascendance of a particular stage of his specialty. His power, in many ways, will replace tradition as the sanction of parenthood. (pp. 38-39) 
Thus, the ideas behind emerging adulthood have been swirling around for some time. As such, this would be an accurate criticism of Arnett, were it not for the fact that he fully recognized the existence of these ideas in his formulation (Arnett, 2000, 2004; Arnett \& Tanner, 2011). What was new, however, was the integration of multiple sources of information into a theory of development during a particular point in the lifespan. Furthermore, the research tradition in sociology was (and still is) to refer to the period as the "transition to adulthood." As Arnett (2004) remarked, referring to it as a "transition” suggests that the period is fleeting and "...leads to a focus on what young people in that age period are becoming, at the cost of neglecting what they are.” (p. 19; italics in original). In contrast, emerging adulthood seeks to understand the psychological experience of young people as they occupy that life space.

2. Emerging adulthood is historically specific. Hendry and Kloep (2007a) warn that it is dangerous to promote a theory of development based on historical trends, fads, or fashions. In their view, theories should be applicable across space and time. This view, however, is hard to defend. Human society has changed dramatically across the world in the last 100 years. These changes have been associated with accompanying changes in how humans function and interact with one another. Here again, Erikson (1968) eloquently states the importance of changing conditions:

A new generation growing up with and in technological and scientific progress as a matter of course will be prepared by the daily confrontation with radically new practical possibilities to entertain radically new modes of thought. This may form a link between a new culture and new forms of society, allowing for ways of balancing specialization with new inner freedom. (p. 38)

If society and individuals therein are changing, do we not also need new theories to understand the changes that have come about? As will be discussed later in the chapter, it is easy to lose sight of the fact that contemporary adolescence in industrialized societies, now taken as a given by researchers, is a relatively recent invention that came about in response to changes in society, most notably compulsory schooling and child labor laws (Hall, 1904; see also Arnett, 2006; but see Schlegel \& Barry, 1991).

3. Emerging adulthood is not a positive time. Arnett (2004) has found that emerging adults tend to have a positive sense about their current situation and the future. They enjoy life and have an optimistic and hopeful view of the future, which is captured in the theory by the age of possibilities. Although Arnett (2004) has viewed this positivity with some skepticism, the theory of emerging adulthood contains a strong positive aura, which has been the source of some criticism. Unlike the other criticisms described here, the issue is not the reality and utility of emerging adulthood itself, but rather how it is conceptualized and portrayed by psychologists. In particular, critics reject Arnett's (2004) optimistic view of emerging adulthood as a time of exploration and opportunity and suggest that the causes and consequences of emerging adulthood are restrictive in nature. Côté (2000, 2006; Côté \& Bynner, 2008) discussed several economic and social factors that lead to a forced emerging adulthood. For example, shifts in labor demands from manufacturing to service had led to restrictions for positive job opportunities absent higher education. This change has resulted in 
higher rates of attendance in higher education, as many emerging adults view college as the only route to a secure, well-paying job. At the same time, the lack of contemporary social norms governing behavior and life choices has led to a diffusion of choice-wandering rather than exploring - which requires greater effort to achieve a stable identity. Taking a somewhat different perspective, Smith (2011) argued that the changes that led to the creation of emerging adulthood have also led to a compromised sense of morality and disengagement from generative aspects of society, such as civic and political involvement. Importantly, neither Côté, Smith, nor any others who share these views makes the claim that emerging adulthood is all negative; rather, they highlight the negative aspects of the life stage to bring balance to the positivity that Arnett made central to emerging adulthood theory.

4. Emerging adulthood only applies to certain people. The argument that emerging adulthood only applies to a certain sector of the world's population is, arguably, the most frequent criticism. The argument is made both in terms of generalizability beyond highly industrialized societies, as well as within those societies. Concerning the latter, emerging adulthood is often argued as a luxury that can only be experienced among those with sufficient means. As such, some critics doubt that youth who occupy marginal social positions - mainly in terms of race/ethnicity, social class, and educational attainment-are emerging adults (Bynner, 2005; Hendry \& Kloep, 2007; 2011; Kloep \& Hendry, 2011). However, Arnett (2000) originally argued that the theory of emerging adulthood could bring greater attention to the developmental experiences of the "forgotten half." Indeed, is his early research Arnett (2004) included youth with diverse social class and educational backgrounds, and he has increasingly devoted attention to the applicability of emerging adulthood across class lines (Arnett \& Schwab, 2012). Who "gets to be” an emerging adult is a site of ongoing inquiry in the literature, and will be discussed in more detail later in the chapter (see also Syed \& Mitchell, 2013).

5. Stages are not good. Nearly since the inception of the field of developmental psychology, there has been a debate about how to conceptualize the course of development (Arnett \& Tanner, 2009). On the one hand, developmental psychology has been dominated by "grand theories" that conceptualized development in terms of a series of universal stages, or discontinuous phases of development (e.g., Freud, Erikson, Piaget, Loevinger, Kohlberg). The opposing view is one that promotes continuous development, in which change is conceptualized as gradual and contextually dependent. Life course theory, life span theory, developmental contextualism, and dynamic systems theories are all examples of theories that conceptualize development as continuous in nature (Baltes, 1987; Elder, 1998; Lerner, 1996; Thelen \& Smith, 1994). Arnett (2000) jumped right into the middle of this simmering debate when he proposed emerging adulthood as a new life stage. Accordingly, this aspect of emerging adulthood has been a source of major criticism.

6. Emerging adulthood is not really a theory. Arnett (2000, 2004, 2012) has always referred to emerging adulthood as a theory. Arguing that it is a theory, rather than a new life stage only, has opened the door for a specific set of criticisms (Hendry \& Kloep, 2007a, 2007b, 2011; Hendry, 2011; Kloep \& Hendry, 2011). The main argument against emerging adulthood being a theory is that it is merely descriptive, rather than explanatory. Arnett and Tanner (2009, 2011) have retorted that a good measure of a theory is the degree to which it is useful 
and generates subsequent research, which they argue emerging adulthood has accomplished. This exchange cuts to the very core of social science research, raising questions about what a theory is and ought to be.

These six criticisms are not purported to represent an exhaustive list, but rather represent my own synthesis of the major challenges that are presented to the theory of emerging adulthood.

Additionally, they are not always so easily separated into distinct criticisms. For example, part of the reason that Hendry and Kloep (2007a) argue against emerging adulthood as a new life stage is that it only applies to certain people. In what follows, I take two of the criticisms - that stages are not good and that emerging adulthood is not really a theory-into much greater depth, as I find them to be the most powerful, far-reaching, and interesting points. In doing so, I also incorporate aspects of the other four criticisms, again highlighting the interconnectedness of the issues.

\section{Stage vs. Process: A Longstanding Debate in Developmental Science}

Hendry and Kloep (2007a), who are among the most visible critics of emerging adulthood, invoke a long-standing debate in developmental science: does development occur in stages or is development a continuous process? One of the major issues they take with emerging adulthood is that it perpetuates stage-like thinking. In this section, I first discuss the ideas of "stages" by considering some well-known developmental stage theories. I then specifically examine emerging adulthood as a stage theory, highlighting some necessary future directions.

Flavell (1963) defined a developmental stage as one "whose qualitative similarities and differences serve as a conceptual landmarks in trying to grasp [a] process” (p. 19). Stages involve four properties: 1) They index qualitative change, in that the differences between successive changes is that of type rather than quantity; 2) They are invariant; development must occur in sequence and individuals may not skip a stage, 3) They are hierarchical, as earlier stages provide the foundation for development to occur at later stages; and 4) They are coherent, with individuals showing integrated functioning across a variety of domains (Flavell, 1963, 1971). Stages of this sort are common defining features of the grand theories of developmental psychology, perhaps none more so than Piaget's (1970) stages of cognitive development (sensorimotor, preoperational, concrete operational, and formal operational). Piaget's theory continues to be a dominant force in research on child development, which can be seen in the large literatures on object permanence, joint attention, and theory of mind, among many others.

Flavell's definition of a developmental stage represents the general thinking about stages in the developmental literature, but it is not the only way of conceptualizing stages. Take, for example, Erik Erikson's (1950) psychosocial theory of development. Erikson specified a lifespan model of development consisting of eight psychosocial tensions. Although frequently described as a stage theory, the theory is not consistent with the definition of a stage provided by Flavell (1963). Erikson's model specifies that all eight tensions reside within individuals at all points in development, but that at different phases of the lifespan one of the tension presents itself as central and in need of resolution. Resolution of one tension provides the foundation for resolution in a subsequent tension, so in this way the tensions are hierarchical. Strictly speaking, however, they are not invariant, as the sequence of identity (tension 5) and intimacy (tension 6) 
clearly illustrates. While identity serves as the foundation for successful intimate relations, individuals can certainly engage in intimacy without having established a reasonably clear identity (Årseth, Kroger, \& Martinussen, 2009; Beyers \& Seiffge-Krenke, 2010). Under Eriksonian theory, that relationship is unlikely to be a successful one, but there is nothing precluding someone from engaging in it or from thinking that intimacy is and should be the prime psychosocial concern of his or her era. Furthermore, disruptions in one of the tensions can result in a revisiting of previously resolved tensions, violating the principle of invariance. Once again considering identity and intimacy, dissolution of an important relationship can cause an individual to revisit his or her identity, even if it had been previously strong (Cookston \& Remy, in press). Thus, for Erikson's theory, there is not a sense of progression that leaves previous issues behind. All resolutions are subject to further scrutiny if the context demands it.

Of course, there is an even broader stage model that is widely used both by researchers and society at large: the age-stage model, as I will call it. ${ }^{1}$ The lifespan has been divided into a series of stages based on certain age markers: prenatal, infancy, toddlerhood, early childhood, middle childhood, adolescence, adulthood, and old age. Just as with the psychological stages of Piaget and Erikson, these stages are mostly arbitrary and can be divided into sub-stages. For example, the prenatal stage consists of three stages (germinal, embryonic, and fetal), and adolescence is often divided into early adolescence, middle adolescence, and late adolescence. With increasing age comes decreasing certainty in the markers of when one age-stage ends and the next one begins. This is due, in part, to the close connection between age-stages and schooling (e.g., early childhood and preschool, adolescence and secondary school) ${ }^{2}$. Beyond secondary school, there are no longer institutionalized parameters to define an age-stage. For this reason, sociologists have long used the "big five" markers of adulthood: leaving home, finishing school, getting a job, getting married, and having children (Settersten, 2011). These markers, however, are much more fluid than the age-graded nature of schooling, and thus there is greater flexibility in how we define age-stages in the third decade of life and beyond.

Understood within this context, adulthood age-stages (i.e., after age 18) are "up for grabs," in that researchers can demarcate them the way they see fit, and there is plenty of room for new theorizing on its subdivisions. Old age is a case in point. The latter years of the lifespan, approximately 65 years and later, has been subject to considerable discussion to understand its heterogeneity. Currently favored subdivisions consists of the young old, old old, and oldest old, but there is no agreement on the use of the terms or where any of the age cutoffs should be located (Binstock, 1992; Neugarten, 1974; Suzman, Willis, \& Manton, 1992). Nevertheless, the ongoing engagement with the topic reflects the belief that there is important heterogeneity in the lifespan beyond age 65, to which a single age-stage not only does disservice, but also discourages ongoing investigation into the heterogeneity.

\footnotetext{
${ }^{1}$ Curiously, despite it widespread use, this stage theory is not often referenced by name and is rarely discussed explicitly in the developmental literature.

${ }^{2}$ Without veering too far off topic, schooling itself is based on cognitive and biological changes, such as the emergence of operational thinking around age 6 and pubertal onset. Schooling, however, it a more visible marker of these changes than are the underlying processes that led to the divisions in the first place. For this reason, schooling and maturation are often confounded in societies with compulsory schooling.
} 
It is within this spirit that Arnett (2000) proposed emerging adulthood as a new agestage. If adolescence ends at age 18, and old age begins at age 65, then a vast portion of the lifespan, 18-65, is left relegated to adulthood. Of course, some make a distinction between young adulthood and midlife (e.g, Lilgendahl, Helson, \& John, 2013; Orth, Trzesniewski, \& Robbins, 2010), but these terms are not used consistently and are arbitrary even by age-stage standards. Arnett's emerging adulthood is a far less arbitrary proposal. In particular, rather than being only a descriptive label for an age-stage, to be used in lieu of 18-29, emerging adulthood has underlying psychological features (e.g., the five pillars). Moreover, its entry and ending points are based on socio-cultural markers that are relevant to that particular point in time. In the U.S., age 18 is the legal definition of adulthood and is the approximate age when young people complete secondary school. The late 20s are the time when young people in the U.S. and other highly industrialized countries now tend to assume the stable adult roles that have historically defined adulthood: marriage, children, stable work, and so on (Arnett, 2011). Thus, emerging adulthood is bookended by socially proscribed developmental milestones.

Stages have long been controversial within developmental psychology. With their focus on qualitative changes and consistent holistic internal structures, stage theories reflect discontinuous views on development. The primary view in contrast to stage-theories is that of continuous development. Whereas stage theories articulate rapid periods of change followed by relative stability, continuous theories emphasize incremental, ongoing development. Continuous development can be irregular-involving the changes and plateaus of stage theories-but such irregularities are not regarded as age-graded, and are more idiosyncratic than normative. There are many different approaches to continuous development, and it is beyond the scope of the current chapter to review them here (see Arnett \& Tanner, 2009, for a partial review in the context of stage theories). My focus in the remainder of this section is on general criticisms of stage theories, both writ large and in terms of emerging adulthood.

One criticism levied at stage theories from proponents of continuous development is the use of sub-stages in their models. As discussed earlier, age-stages such as prenatal and adolescence are frequently divided up into substages. This is also the case for psychological stages. Piaget's sensorimotor stage is a prime example. The first stage in Piaget's model, the sensorimotor stage begins at birth and proceeds until approximately age 2, or the time in which the child is capable of internal thought. However, to explain the changes that occur during the stage - which by definition involves little change-Piaget developed six sub-stages for those two years (e.g., primary circular reactions, secondary circular reactions). The sub-stages reflect the increasing ability to coordinate thought and action, which is the primary developmental task of the sensorimotor stage (hence the "sensorimotor" label). As sub-stages are added and refined, the theory begins to resemble a continuous theory in which development is constant, in lieu of identifiable long stretches of stability. This issue of sub-stages in emerging adulthood is important, and few researchers have addressed it directly. There are occasional references to early, middle, and late emerging adulthood, suggesting that researchers find emerging adulthood itself to be too broad (e.g., Tanner, 2006). The definitions of these sub-stages, however, are not clear and have been scantly investigated.

In terms of emerging adulthood theory specifically, one of the salient arguments against it is that it is both inappropriate and dangerous to create a new life stage that is not universal. The 
universality criticism applies at two levels: within and between countries. Within countries, it has been argued that emerging adulthood only applies to those who are privileged enough to enjoy it, often defined as white, middle-class, and college students-despite the fact that Arnett's (2004) original research included ethnically-diverse emerging adults who were not in college. There are little data, however, to back up these claims, and the argument is much more complex than it may seem on its surface (see Arnett \& Tanner, 2011; Syed \& Mitchell, 2013). We took up the issue of race/ethnicity in-depth in Syed and Mitchell (2013), so here I will briefly focus on class and college-more specifically how they intersect.

The equating of middle-class, college students, and privilege/luxury relies on outdated stereotypes. What are often referred to as "traditional” college students — attending 4-year residential college straight out of secondary school-have become the minority in the U.S. Indeed, nearly three-fourths of college students are considered "non-traditional" in some way, due to increased age, attending community college or vocational school, heavy paid work commitments, being a parent, and so on (Waters, Carr, \& Kefalas, 2011). Thus, assuming that all college students are privileged is based more on myth than reality. To be sure, there are severe disparities in college attendance and graduation by social class (Haskins, Holzer, \& Lerman, 2009). Nevertheless, there are youth from low-income backgrounds who attend college, just as there are those from wealthy families who do not, indicating the need to examine how emerging adulthood applies in terms of class and college, both separately and as they interact. Unfortunately there are few empirical studies that have done this.

Critics also argue that emerging adulthood is not apparent around the world. Indeed, from the beginning Arnett (2000) indicated that emerging adulthood was a new life stage in highly industrialized countries, where the social and economic conditions were ripe for it (see Arnett, 2011 for a more recent and nuanced discussion). Thus, there is broad agreement that, at least between countries, emerging adulthood is not universal. But does this fact disqualify it from being a developmental age-stage? Those of you readers who answer in the affirmative may want to take a closer look at the age-stage immediately preceding emerging adulthood: adolescence. Adolescence is not experienced equally across the world, and there is debate about whether or not adolescence exists in all cultures (Schlegel \& Barry, 1991). For those cultures in which there is a period of adolescence, there is tremendous variation in the form and function of adolescence. Adolescence is viewed as a time of preparation; in which biological and social transitions deliver the child into adulthood. Thus, the definition of adulthood has implications for understanding the function of adolescence. In industrialized countries adulthood is often marked by independence whereas in traditional cultures it is often marked by marriage. These different markers of adult status suggest that the developmental tasks of adolescence will be different for adolescents living in these different cultures, despite the fact that both are considered "adolescents.” Despite the rather stark culture differences in adolescence, there are few serious scholarly attempts to question adolescence as a life stage. Rather, attention is devoted to variations in how adolescence is conceptualized and experienced. Indeed, Arnett (2011) draws upon the scholarly work on adolescence in his development of the concept of "many emerging adulthoods." Rather than a singular understanding of emerging adulthood, Arnett argues for emerging adulthood as a cultural theory, in which emerging aduulthood is shaped by social class, ethnicity, gender, religion, economics, and so on. While the general outline of emerging adulthood may be the same around the world, the content of it will vary both within and between cultures. 
In a related vein, Hendry and Kloep (2007a) state that one cannot simply invent a new life stage. This statement is problematic for two reasons. First, as discussed earlier, age-stages are mostly arbitrary, leaving room for new ones to be invented and old ones discarded. Second, this view is historically myopic. Indeed, many have argued that adolescence, as it is understood in contemporary industrialized societies, was invented around end of the $19^{\text {th }}$ century-very recently in phylogenetic terms. Like emerging adulthood, the emergence of adolescence has been linked to industrialization, namely more restrictive child labor laws and the establishment of compulsory schooling. As Schlegel and Barry (1991) argue, the links to economic development are not adequate to explain the apparent universal presence of adolescents. However, it can help explain some of the cultural variations in adolescence around the world.

It is also important to note that the definition of adolescence has changed over time, highlighting the fluidity and social dependency of age-stages. Hall (1904) defined adolescence as ages $14-24$. These ages may seem arbitrary and strange to the $21^{\text {st }}$ century reader, but they made sense at Hall's time: 14 was the average age of puberty and 24 was the average age of marriage. Indeed, adolescence has always been defined as beginning in biology and ending in culture. The problem is that both biology and culture change over time. Pubertal timing has been decreasing steadily since Hall's time, bringing the onset of adolescence down with it. At the same time, compulsory school and legal ages of adulthood have provided a fixed ending point for adolescence. Thus, the common age definition of adolescence ends up being 10-18. This definition is rather different than Hall's 14-24, and bears no resemblance to anything that has come before it. Thus, over the past 100 years, a new life stage has most certainly evolved.

The variation in the timing of adolescence raises an important point for emerging adulthood. Although the average age of puberty is 10, the normative range is 2 years around that point estimate, so 8-12. For example, a 12 year old boy who has not yet begun puberty is likely surrounded by a large number of friends and schoolmates who have begun puberty. Thus, although our 12-year old is not yet an adolescent, he is occupying the socially defined period of adolescence.

Syed and Mitchell (2013) recently articulated the importance of making a distinction between emerging adulthood and emerging adults. Emerging adulthood is the label Arnett (2000) developed to describe a new stage of the life span, and outlines the normative social and psychological conditions that define it. In contrast, emerging adults is the term for the people themselves - not the age-stage. Emerging adults (the people) occupy emerging adulthood (the period). This distinction is important, as it highlights the interactions of the psychological and sociological levels of analysis within emerging adulthood theory. As described earlier, there is little debate that the timing and meaning of adulthood has changed in many parts of the world. In other words, I would say there is little debate about the sociological phenomenon of emerging adulthood $^{3}$. However, there are many questions about emerging adults, namely, who is included and excluded from participating in emerging adulthood? As previously discussed, not enough is known to properly answer this question. What is important, however, is that even if some youth are identified as "not emerging adults" it does not follow that there is no emerging adulthood. Emerging adulthood, as a sociological phenomenon, is a social and cultural context that must be

\footnotetext{
${ }^{3}$ There are, however, debates on how the changes occurred and what they mean for youth (e.g., Bynner, 2005; Côté, 2006).
} 
navigated by young people who pass through it. In other words, even if an individual is not an emerging adult, he/she develops within the context of emerging adulthood, which would likely have implications for said development. Unfortunately, these proposals have not been examined empirically.

So, is emerging adulthood a new life stage? In my discussion, I attempted to increasingly blur the lines between stage (discontinuous) approaches and process (continuous) approaches by highlighting variations within normative and meaningful structures (see also Arnett, 2011). Can we have it both ways? Pepper's (1942) seminal World Hypotheses suggests that we may not be able to, but that the two approaches may not be as dissimilar as they seem. Pepper suggested that all scientific inquiry could be located through four non-reducible root metaphors: formism, mechanism, organicism, and contextualism. Hendry and Kloep (2007) argue strongly for dynamic systems theory, which seeks understanding within complex, interdependent, and chaotic systems. In this way, they are invoking contextualism, the root metaphor that captures dynamic and idiosyncratic processes. In contrast, emerging adulthood, as a stage theory, flows from a formistic root metaphor, indicating that development can be isolated into well-defined and unique categories or forms.

On the one hand, contextualism and formism are opposites in terms of how they handle complexity; embracing it versus reducing it, respectively (Cooper, 1987). On the other, they are both dispersive theories, in that they reject order and determinism and "take data as they come," and are fundamentally concerned with complex processes. This similarity in orientation can be seen in the fact that many theorists have used stage concepts, including Piagetian notions of assimilation and accommodation (e.g., Kunnen and Metz, in press) or challenges as the driver of development (Hendry \& Kloep, 2007a), while advocating for dynamic systems (see also DeYoung, 2010 for a similar approach within personality psychology). Indeed, Hendry (2011) recently conceded that he and Arnett mostly agree on the underlying psychological processes that are occurring, and that his main point of contention was the age-stage label. This discussion of root metaphor and theory now assumes that emerging adulthood is indeed a theory. In the next section, I take a much closer look at whether or not that is the case.

\section{What is Theory? Is Emerging Adulthood a Theory?}

Is emerging adulthood truly a theory? To address this question, we must first back up a bit and ask a broader question: what is a theory? As it happens, for several reasons this not such a simple question to address.

First, the definition and purpose of theory is disciplinary. Iser (2006) draws a clear distinction between scientific theory and humanistic theory. ${ }^{4}$ In general, scientific theories are oriented towards making predictions that will ultimately lead to the development of laws. In contrast, humanistic theories are oriented towards developing greater understandings, with the ultimate goal of developing a functional metaphor that represents the phenomenon. Scientific

\footnotetext{
${ }^{4}$ Iser refers to scientific theories as "hard-core” and humanistic theories as "soft.” I intentionally avoid this language, as it reinforces the existing hierarchy of hard-science and soft-science, which implies that rigor is a matter of quantity rather than quality.
} 
theories are discarded through the process of falsification, whereas humanistic theories are discarded because scholars are no longer interested in using them.

All theories emphasize explanation. The fundamental difference between scientific and humanistic theories, from which all other differences follow, is how explanation is conceptualized, and what it leads to. Scientific theories are meant to generate predictions, whereas humanistic theories are meant to develop understandings, and have little, if any, interest in prediction. Another way to think about this distinction in the use of theory is to use language that should be familiar to most researchers in the social sciences: deductive and inductive inference.

Deductive inference is a top-down process wherein a set of propositions (e.g., data, speculation) is used to develop a testable hypothesis. The results of the test are evaluated in terms of the degree to which they are consistent with the hypothesis. Prediction plays a critical role in deductive inference, as the nature of the prediction that is made by the researcher dictates the type of data that are collected. The origins of the prediction are of little consequence, so long as the data are proper tests of the hypothesis (Popper, 1959).

Inductive inference, in contrast, is a bottom-up process wherein data are gathered and subsequently organized into a coherent system. Prediction does not play a role at all in inductive inference, as that is simply not a goal of the approach. Observations are collected in order to develop a deeper understanding of a phenomenon. Thus, as with humanistic theories, the goal of inductive inference is understanding, not prediction. From a postpositivistic scientific perspective, there are clear limits to inductive inference, most notably that theories developed inductively rely on verifiability and have not been subject to falsifiability (Popper, 1959).

In what is perhaps a reflection of psychology's position within the so-called hierarchy of science (Fanelli, 2010; Simonton, 2006), psychological research makes use of a curious combination of these two ways of doing theory (Meehl, 1990). Indeed, this metatheoretical diversity is what underlies one of the most contentious and ongoing debates among psychological researchers: the question of quantitative and qualitative methods (Lyons, 2009). Many researchers do not have a conscious awareness of this alignment, and thus fail to realize that they are arguing about approaches that are, in fact, complementary. But all of that is a topic for a different chapter. What I am concerned with here is how, no matter if a theory was developed deductively or inductively, most psychological researchers want to have certain shared qualities that make the theory useful.

Usefulness is a concept that has been long prized in the social sciences. Take, for example, two oft-cited dictums (paraphrased): there is nothing so practical as a good theory (Lewin, 1951) and all models are wrong, but some are useful (Box, 1976). But how do we determine if a theory is useful? The usefulness of a theory should ultimately be judged by the degree to which it can inform why elements of a system are inter-related. In doing so, it should have five key features (Gelso, 1996): descriptive, delimiting, generative, integrative, parsimonious. I will discuss each of these in turn. 
A theory must be descriptive, with strong understanding of the phenomenon in question. The description should be deep enough to allow for explanation, which is the true goal of comprehensive descriptiveness. Although explanation is the desired end state, description is highlighted because it is only through describing the psychological phenomenon that explanations can be developed. Furthermore, explanation is best understood as the product of all five qualities of theories described here, as it is the basis for prediction (science) or understanding (humanities).

A theory must also be delimiting, in that the terrain that is and is not covered by the theory should be clear. Delimiting a theory sets the boundaries on what falls under the purview of the theory, with clear boundaries leading to stronger predictions and less frequent overextensions. As I have written elsewhere (Syed, Azmitia, \& Cooper, 2011), stereotype threat (Steele, 1997) is a highly visible example of a theory that has been over-extended. Using a theory to generate hypotheses that are outside of the scope of the theory leads to a murky cumulative record, and ultimately compromises the utility of the theory.

Related to the principle of usefulness, a theory should be generative, in that it inspires subsequent research. A useful theory is one that is subsequently adopted by other researchers and evaluated extensively, thereby refining the original theory. A theory that does not generate further scholarly work is not particularly useful.

A critical aspect of theories is that they are integrative, in that they pull many different pieces of information together into a coherent system. Ideally, this integration includes laws, principles, and the results of tested hypotheses, as well as other observations and intuitions. In critiquing Markus and Kitayama’s (1991) theory of independent and interdependent selfconstruals, Matsumoto (1999) noted that one of the reasons for its widespread adoption is that it appeared to account for an enormous amount of data. That is, it integrated a variety of disparate findings in the areas of cognition, emotions, and behaviors into a simple model with strong face validity.

Finally, the integrativeness of a theory should be balanced out with parsimony. That is, a theory should include only those postulations that are required for its usefulness, and nothing more. Following Occam's Razor, when two accounts have equal predictive power, the simpler one should always be adopted. This approach dovetails with the modeling revolution in data analysis (Rodgers, 2010), in which analysts seek to develop the simplest possible model that can account for the greatest amount of the original variance. A useful theory is one that finds an optimal balance between integrativeness and parsimony.

Descriptive, delimiting, generative, integrative, parsimonious. These five features of a good theory are, of course, interrelated. As just discussed, a theory's integrativeness must be considered in relation to how parsimonious it is. Additionally, a theory is only likely to generate further research if there is a sufficient amount of descriptive data that outlines what the theory actually is, and is sufficiently delimited so that others know to whom, what, and when the theory pertains. Moreover, these five features are not the only dimensions through which a theory might be evaluated. Nevertheless, they provide a useful heuristic for taking a deeper and more systematic look at whether an ostensible social science "theory" is in fact what it claims to be. 
Part of the sloppiness among the use of the term theory is because, in general, psychological theories are quite weak (LeBel \& Peters, 2011) and have very few of the features described above. Additionally, the distinction between theory and hypothesis is not always clear. Theories are abstract, general, and generative. Hypotheses are concrete, specific, and testable. Theories should be used to generate hypotheses to be tested, the results of which then lead to a modification of theory.

The use of theory is also not uniform across disciplines of psychology. Social psychology, for example, relies on myriad micro-theories that often bear more resemblance to hypotheses (see Van Lange, Kruglanski, \& Higgins, 2011). In contrast, developmental psychology is dominated by a few old grand theories, such as Piaget's (1970) and Vygotsky's (1978) theories of cognitive development, and psychoanalytic theory and its derivatives (e.g., identity theory, Erikson, 1968; attachment theory, Bowlby, 1969). Still yet, personality psychology, with its heavy reliance on trait taxonomies, is mostly atheoretical (DeYoung, 2010). At the same time, there are other organizing systems that are called theories but do not suggest any testable predictions. These include Bronfenbrenner's (1979) ecological model, McAdams' (2013) three levels of personality, and intersectionality (Cole, 2009). These models are best conceptualized as frameworks that can serve as structures for housing different theories (see Syed, 2010). The point of all of this is that establishing what is and is not a theory is not a straightforward task. Given this somewhat lengthy treatment of what theory is, we can now properly turn to the question at hand: is emerging adulthood a theory? To do so, we must consider emerging adulthood in terms of the five aspects of theory.

\section{Is it Descriptive?}

The degree to which emerging adulthood is descriptive is an interesting topic, one that has featured prominently in the debate (Arnett, 2007; Hendry \& Kloep, 2007a). The debate, however, has not been about whether or not emerging adulthood is descriptive. Instead, the fact that is descriptive has been used as the basis for which it is not a theory. For example:

A theory needs to be more than a description of characteristics and behaviours of particular (relatively privileged?) groups in relatively wealthy Western societies.... the construct of emerging adulthood is a description and does not especially advance our knowledge and understanding of human development” (Hendry, 2009, pp. 1-2).

Indeed, emerging adulthood is largely a collection of characteristics and behaviors of the populations that have been observed. It describes what emerging adults do, what they think, how they feel, and so on. In making his point, however, Hendry (2009) seemingly derides description, rather than seeing it as a necessary component of a theory and the route towards explanation. In a subsequent comment, Hendry (2009) states, “Age, like other structural variables such as gender, social class, or ethnicity, may predict, but does not explain, developmental phenomena.” (p. 2; italics added).

Taking these comments together, Hendry appears to have put his theoretical cart before the horse. Scientific research involves five steps that, to be successful, must occur in a particular order: observation, description, explanation, prediction, and control. Engaging in any of these 
steps before having a firm footing on the previous ones will almost guarantee failure. As an obvious example, it is mighty difficult to describe something that one has not yet observed. Description is critical to the development of any theory. Without description, there can be no explanation. It is obviously a matter of opinion, but I would argue that most psychological theories — or even social science theories—sit at the interface of description and explanation. We are very good at description, but this has yet to translate into solid and consistent explanation. So where does emerging adulthood fit in? I agree with Hendry that is almost entirely descriptive. My point of departure, however, is that I view that as a good thing (at least for now).

\section{Is it Delimiting?}

Despite what some of the critics have asserted, Arnett (2000) was clear from the beginning that emerging adulthood was not to be considered a universal life stage: "Is emerging adulthood a period of life that is restricted to certain cultures and certain times? The answer to this question appears to be yes.” (Arnett, 2000, p. 477; italics in original). He goes on, "emerging adulthood would be most likely to be found in countries that are highly industrialized or postindustrial.” (p. 478). Thus, the theory of emerging adulthood in its original form contained some boundary conditions (Arnett, 2011).

Nevertheless, delimiting the theory has been one of the major focuses of its critics. In particular, some have asserted that, even within highly industrialized societies, it is only the privileged young people who "get" to experience it. Here privilege is typically defined in terms of race/ethnicity, social class, and educational attainment. Despite the reasonable criticisms, there have been few careful analyses to demonstrate that certain sectors of the populations are shut out from emerging adulthood (Arnett and Tanner, 2011). Syed and Mitchell (2013) took up the task of reviewing the literature on race, ethnicity, and emerging adulthood and, not surprisingly, concluded that the degree to which emerging adulthood generalizes across ethnic and racial lines is complicated and depends on a number of factors. The findings of the Clark University Poll of Emerging Adults (Arnett \& Schwab, 2012), a survey of over 1,000 emerging adults across the U.S., suggested that there were few differences in endorsement of emerging adulthood criteria across social classes (defined as mother's educational attainment). Emerging adults whose mothers' educational attainment was high school or less reported having greater financial challenges to fund the education they desired, viewed their lives as less fun and exciting, and reported greater depressive symptoms. At the same time, they were more positive about their futures, and were more likely to report that they expect their lives to be better than those of their parents.

As discussed previously it is important to not conflate social class with participation in higher education. Like direct comparison by social class, there are relatively few direct comparisons between college and non-college goes. Hendry and Kloep (2010) provided an analysis of heterogeneity of emerging adulthood among those who were not in college, but they did not include a college-going comparison group. In contrast, a recent analysis compared four groups of emerging adults: traditional college students at a four-year university, first-generation college students (i.e., first in their family to attend college), technical college students, and nonstudents (Mitchell, Syed, McLean, \& Wood, 2013). The findings indicated that there were differences among the four groups but they were not widespread, primarily in terms of self- 
focus, other-focus, and possibilities. Based on the findings, Mitchell et al. suggested that emerging adulthood should be considered as a continuous range, a matter of degree, rather than either/or.

In general, there has been a degree of delimitation to theory of emerging adulthood, but there is a pressing need for much more research on this topic. Despite the diversity of Arnett (2004) original sample, the majority of research on emerging adulthood has been conducted with college students, and therefore the claim that it does not generalize to non-college populations has been assertion rather than based on data. The truth is, we do not really know either way.

\section{Is it Generative?}

This may be emerging adulthood's strongest footing as a theory. As Arnett and Tanner (2011) noted, Arnett's 2000 American Psychologist article had been cited over 1700 times as of September 2010. Three years later (January 2014), the number of citations is over 4000 (according to Google Scholar). There is now a Conference on Emerging Adulthood that held its $6^{\text {th }}$ biennial meeting in 2013, the Society for the Study of Emerging Adulthood officially launched in 2011 and has over 400 members worldwide, and in 2013 the new journal Emerging Adulthood was launched as the flagship journal of the society and is poised to become the premier outlet of scholarship pertaining to the third decade of life. Thus, there is little doubt that the theory of emerging adulthood has been generative. As Arnett and Tanner (2011) remark, "The key question for any theory should be, does it inspire, new knowledge and research?...For emerging adulthood, the answer is unequivocally yes.” (p. 124). While I agree with the conclusion, I disagree that generativity is the key question. It is, rather, one question among many that must be examined.

\section{Is it Integrative?}

Emerging adulthood does integrate a number of different observations, on historical, social, and individual levels. The conditions for emerging adulthood are located in economic (shift to services, information, and technology) and societal (compulsory schooling, birth control) changes. The five pillars themselves represent the different aspects of youth's lives that have been pulled together. Instability covers frequent changes in several domains, particularly where people live, the jobs they hold, and their romantic relationships. Possibilities capture an affective orientation towards the future. Self-focus highlights how emerging adults establish priorities in their busy lives. Feeling in-between signifies how they think about the meaning of adulthood. Finally, identity exploration, the grand pillar, represents a broad tendency to view the present as unsettled, developing, and emerging. Thus, it would seem that emerging adulthood integrates a broad variety of observations.

\section{Is it Parsimonious?}

The degree to which emerging adulthood is appropriately parsimonious is difficult to judge. It may depend on what aspect of emerging adulthood is being considered. On the one hand, a theory of development that is meant to capture the experiences of a large portion of the world is extremely parsimonious - perhaps too much, as some have suggested (Hendry \& Kloep, 
2007a). As described previously, however, parsimony must be understood in the context of the information that it is trying to integrate. Emerging adulthood is, in fact, much more than just a label applied to an age-stage. Indeed, there are psychological experiences that undergird it. While the five pillars have face validity, it is unclear just how necessary they all are to the theory. For example, it could be that identity exploration serves as the broader psychological construct that accounts for the instability and possibilities. That is, are they simply behavioral and affective manifestations, respectively, of the identity development process? These are the types of questions that have not yet been directly evaluated.

\section{So, is it a Theory?}

As should be clear from the preceding, answering the question of whether or not emerging adulthood is a theory is not an easy task. It certainly is strong in some aspects, namely generativity and integrativeness. Other aspects are more questionable, such as delimiting and parsimonious. It is descriptive, but not comprehensively enough so that we are very close to having reasonable explanations for what has been observed. Indeed, age has served as the primary explanation for emerging adulthood (Arnett et al., 2011). Age, like other demographic constructs (e.g.. ethnicity, gender), has little to no explanatory power, but serves as a proxy for other, more dynamic processes. What are these processes for emerging adulthood? The five pillars? At this point, the answers to these questions are not well known, and investigating them will be critical to understanding the theory of emerging adulthood.

So, is emerging adulthood a theory? In the debates between Arnett and Hendry, the two (and colleagues) argued about its theoretical merits using different criteria; generativity and description/explanation, respectively. As it turns out, for the most part they are both correct. Emerging adulthood has been incredibly generative, but does not have a lot of explanatory power. Thus, I would say that it is a theory in development-emerging perhaps-but that much more work needs to be done.

\section{Rejoinder: But is it Nonsense?}

Nonsense seems a bit harsh, but as noted at the outset of this chapter, I am not exactly an impartial observer. Despite my strong involvement with the emerging adulthood enterprise, my mission has not been to bolster emerging adulthood, but rather to take a deeper look at some of the criticisms that have been made as assertions of fact. My colleagues and I have approached that task both conceptually (Syed \& Mitchell, 2013) and empirically (Mitchell et al., 2013). The results of these analyses suggest that statements such as "emerging adulthood does not apply to X” are both unhelpful and unsupported. Indeed, yes/no evaluations of emerging adulthood are unlikely to be useful for the development (or dismissal) of the theory. Rather, questions of quality - for whom, under what conditions, and to what to degree-will assist with theoretical development, particularly concerning the boundary conditions of the theory. Arnett (2011) himself has increasingly endorsed this view, writing about the "many emerging adulthoods" that can be seen within and between cultures. It may be that emerging adulthood is "a description of characteristics and behaviours of particular (relatively privileged?) groups in relatively wealthy Western societies” (Hendry, 2009, p. 1), but I have yet to see any convincing data that support (or refute) such a claim. 


\section{References}

Arnett, J. J. (1994). Are college students adults? Their conceptions of the transition to adulthood. Journal of Adult Development, 1(4), 213-224.

Arnett, J. J. (1997). Young people's conceptions of the transition to adulthood. Youth \& Society, 29(1), 3-23.

Arnett, J. J. (1998). Learning to stand alone: The contemporary American transition to adulthood in cultural and historical context. Human Development, 41(5-6), 295-315.

Arnett, J. J. (2000). Emerging adulthood: A theory of development from the late teens through the twenties. American Psychologist, 55(5), 469-480.

Arnett, J. J. (2004). Emerging adulthood: The winding road from late teens through the twenties. New York: Oxford University Press.

Arnett, J. J. (2006). Emerging adulthood: Understanding the new way of coming of age. In J. J. Arnett \& J. L. Tanner (Eds.), Emerging adulthood: Coming of age in the $21^{\text {st }}$ century (pp. 3-20). Washington, DC: American Psychological Association.

Arnett, J. J. (2007a). Emerging adulthood: What is it, and what is it good for? Child Development Perspectives, 1(2), 68-73.

Arnett, J. J. (2007b). Emerging adulthood, a 21st century theory: A rejoinder to Hendry and Kloep. Child Development Perspectives, 1(2), 80-82.

Arnett, J.J. (2011). Emerging adulthood(s): The cultural psychology of a new life stage. In L.A. Jensen (Ed.), Bridging cultural and developmental psychology: New syntheses in theory, research, and policy (pp. 255-275). New York: Oxford University Press.

Arnett, J. J. (2012). New horizons in research on emerging and young adulthood. In A. Booth, S. L. Brown, N. S. Landale, W. D. Manning, \& S. M. McHale (Eds.), Early adulthood in a family context (pp. 231-244). Springer : New York.

Arnett, J. J. (2013). The evidence for generation we and against generation me. Emerging Adulthood, 1(1), 5-10.

Arnett, J. J., Kloep, M., Hendry, L. B., \& Tanner, J. L. (2011). Debating emerging adulthood: Stage or process? New York: Oxford University Press.

Arnett, J. J., \& Schwab, J. (2012). The Clark University Poll of Emerging Adults: Thriving, struggling, and hopeful. Worcester, MA: Clark University.

Arnett, J. J., \& Taber, S. (1994). Adolescence terminable and interminable: When does adolescence end? Journal of Youth and Adolescence, 23(5), 517-537.

Arnett, J. J., \& Tanner, J. L. (Eds.). (2006). Emerging adults in America: Coming of age in the 21st century. Washington, DC: American Psychological Association.

Arnett, J. J., \& Tanner, J. L. (2009). Toward a cultural-developmental stage theory of the life course. In. K. McCartney, \& R. A. Weinberg (Eds.), Experience and development: A festschrift in honor of Sandra Wood Scarr (pp. 17-38). New York: Psychology Press.

Arnett, J. J., \& Tanner, J. L. (2011). Themes and variations in emerging adulthood across social classes. In Arnett, J. J., Kloep, M., Hendry, L. B., \& Tanner, J. L., Debating emerging adulthood: Stage or process? New York: Oxford University Press.

Årseth, A. K., Kroger, J., Martinussen, M., \& Marcia, J. E. (2009). Meta-analytic studies of identity status and the relational issues of attachment and intimacy. Identity: An International Journal of Theory and Research, 9(1), 1-32.

Baltes, P. B. (1987). Theoretical propositions of life-span developmental psychology: On the dynamics between growth and decline. Developmental Psychology, 23(5), 611. 
Beyers, W., \& Seiffge-Krenke, I. (2010). Does identity precede intimacy? Testing Erikson's theory on romantic development in emerging adults of the 21st century. Journal of Adolescent Research, 25, 387-415.

Binstock, R. H. (1992). The oldest old and" intergenerational equity." In R. M. Suzman, D. P. Willis, \& K. G. Manton (Eds.), The oldest old (pp. 394-417). New York: Oxford.

Bowlby, J. (1969). Attachment and loss v. 3 (Vol. 1). Random House.

Box, G. E. (1976). Science and statistics. Journal of the American Statistical Association, 71(356), 791-799.

Bronfenbrenner, U. (1979). The ecology of human development. Cambridge, MA: Harvard University Press.

Bynner, J. (2005). Rethinking the youth phase of the life-course: The case for emerging adulthood? Journal of Youth Studies 8(4), 367-84

Cole, E. R. (2009). Intersectionality and research in psychology. American Psychologist, 64(3) 170-180.

Cookston, J. T., \& Remy, L. (in press). Who am I if we're not us? Divorce and identity across the lifespan. In K. C. McLean \& M. Syed (Eds.), The Oxford handbook of identity development. New York: Oxford.

Cooper, C. R. (1987). Conceptualizing research on adolescent development in the family: Four root metaphors. Journal of Adolescent Research, 2(3), 321-330.

Côté, J. E. (2000). Arrested adulthood: The changing nature of maturity and identity. NYU Press.

Côté J. (2006) Emerging adulthood as an institutionalized moratorium: Risks and benefits to identity formation. In J. J. Arnett \& J. L. Tanner (Eds) Emerging adults in America: Coming of age in the $21^{\text {st }}$ century. Washington, DC: American Psychological Association.

Cote, J., \& Bynner, J. M. (2008). Changes in the transition to adulthood in the UK and Canada: the role of structure and agency in emerging adulthood. Journal of Youth Studies, 11(3), 251-268.

DeYoung, C. G. (2010). Toward a theory of the Big Five. Psychological Inquiry,21(1), 26-33.

Elder, G. H. (1998). The life course as developmental theory. Child Development, 69(1), 1-12.

Erikson, E. H. (1950). Childhood and society. New York: W W Norton \& Co.

Erikson, E. (1968). Identity, Youth and Crisis. New York, NY: Norton.

Fanelli, D. (2010). "Positive" results increase down the hierarchy of the sciences. PLoS One, 5(4), e10068.

Flavell, J. H. (1963). The developmental psychology of Jean Piaget. New York: D. Van Nostrand Co.

Flavell, J. H. (1971). Stage-related properties of cognitive development. Cognitive Psychology, 2(4), 421-453.

Gelso, C. J. (1996). Applying theories in research: The interplay of theory and research in science. In F. Leong \& J. Austin (Eds.), The psychology research handbook: A primer for graduate students and research assistants (pp. 359-368). Thousand Oaks, CA: Sage.

Hall, G. S. (1904). Adolescence: Its psychology and its relations to physiology, anthropology, sociology, sex, crime, religion and education. Englewood Cliffs, NJ: Prentice-Hall.

Hartmann, D., \& Swartz, T. T. (2006). The new adulthood? The transition to adulthood from the perspective of transitioning young adults. Advances in Life Course Research, 11, 253286. 
Haskins, R., Holzer, H., \& Lerman, R. (2009). Promoting economic mobility by increasing postsecondary education. Economic Mobility Project. Retrieved from www.pewcharitabletrusts.org

Hendry, L. B. (2008) A letter from Lucifer. Human Development, 51,1-3.

Hendry, L. B. (2011). Part III: Leo B. Hendry: “As John McEnroe used to say...”. In Arnett, J. J., Kloep, M., Hendry, L. B., \& Tanner, J. L., Debating emerging adulthood: Stage or process? New York: Oxford University Press.

Hendry, L. B., \& Kloep, M. (2007a). Conceptualizing emerging adulthood: Inspecting the emperor's new clothes? Child Development Perspectives, 1(2), 74-79.

Hendry, L. B., \& Kloep, M. (2007b). Redressing the emperor!--A rejoinder to Arnett. Child Development Perspectives, 1(2), 83-85.

Iser, W. (2006). How to do theory. Malden, MA: Blackwell Publishers.

Kloep, M., \& Hendry, L. B. (2011). A systemic approach to the transitions to adulthood. In Arnett, J. J., Kloep, M., Hendry, L. B., \& Tanner, J. L., Debating emerging adulthood: Stage or process? New York: Oxford University Press.

Kunnen, E. S., \& Metz, M. (in press). Commitment and exploration: The need for a developmental approach. In K. C. McLean \& M. Syed (Eds.), The Oxford handbook of identity development. New York: Oxford.

LeBel, E. P., \& Peters, K. R. (2011). Fearing the future of empirical psychology: Bem's (2011) evidence of psi as a case study of deficiencies in modal research practice. Review of General Psychology, 15(4), 371.

Lerner, R. M. (1996). Relative plasticity, integration, temporality, and diversity in human development: A developmental contextual perspective about theory, process, and method. Developmental Psychology, 32(4), 781.

Lewin, K. (1951) Field theory in social science; selected theoretical papers. D. Cartwright (Ed.). New York: Harper \& Row.

Lilgendahl, J. P., Helson, R., \& John, O. P. (2013). Does ego development increase during midlife? The effects of openness and accommodative processing of difficult events. Journal of Personality, 18, 403-416.

Lyons, A. (2009). Is the American Psychological Association ready to accept a division for qualitative inquiry? The Humanistic Psychologist, 37, 101-107.

Markus, H. R., \& Kitayama, S. (1991). Culture and the self: Implications for cognition, emotion, and motivation. Psychological Review, 98(2), 224-253.

Matsumoto, D. (1999). Culture and self: An empirical assessment of Markus and Kitayama's theory of independent and interdependent self-construal. Asian Journal of Social Psychology, 2(3), 289-310.

McAdams, D. P. (2013). The psychological self as actor, agent, and author. Perspectives on Psychological Science, 8(3), 272-295.

Meehl, P. E., (1990). Why summaries of research on psychological theories are often uninterruptable. Psychological Reports, 66, 195-244.

Mitchell, L. L., Syed, M., McLean, K. C., Wood, B. (2013). The generalizability of emerging adulthood theory across educational groups. Manuscript submitted for publication.

Neugarten, B. L. (1974). Age groups in American society and the rise of the young-old. The Annals of the American Academy of Political and Social Science, 415(1), 187-198. 
Orth, U., Trzesniewski, K. H., \& Robins, R. W. (2010). Self-esteem development from young adulthood to old age: a cohort-sequential longitudinal study. Journal of Personality and Social Psychology, 98(4), 645.

Pepper, S. C. (1942). World hypotheses: A study in evidence. Berkeley, CA: University of California Press.

Piaget, J. (1970). Piaget's theory. In P. Mussen (Ed.) Handbook of child psychology, Vol.1. New York: Wiley,

Popper, K. R. (1959). The logic of scientific discovery. London: Hutchinson.

Rodgers, J. L. (2010). The epistemology of mathematical and statistical modeling: a quiet methodological revolution. American Psychologist, 65(1), 1-12.

Sagan, C. (1980). Cosmos. New York: Ballantine Books.

Schlegel, A., \& Barry, H. Ill (1991). Adolescence: An anthropological inquiry. New York: Free Press.

Settersten, Jr., R. A. (2011). Becoming adult: Meanings and markers for young Americans. In M. C. Waters, P. J. Carr, M. J. Kefalas, \& J. Holdaway (Eds.), Coming of age in America: The transition to adulthood in the twenty-first century. Berkeley: University of California Press.

Simonton, D. K. (2006). Scientific status of disciplines, individuals, and ideas: Empirical analyses of the potential impact of theory. Review of General Psychology, 10(2), 98.

Smith, C. (2011). Lost in transition: The dark side of emerging adulthood. New York: Oxford University Press.

Steele, C. M. (1997). A threat in the air: how stereotypes shape intellectual identity and performance. American Psychologist, 52(6), 613.

Suzman, R. M., Willis, D. P., \& Manton, K. G. (Eds.). (1995). The oldest old. New York: Oxford University Press.

Syed, M. (2010). Disciplinarity and methodology in intersectionality theory and research. American Psychologist, 65(1), 61-62.

Syed, M., Azmitia, M., \& Cooper, C. R. (2011). Identity and academic success among underrepresented ethnic minorities: An interdisciplinary review and integration. Journal of Social Issues, 67(3), 442-468.

Syed, M., \& Mitchell, L. L. (2013). Race, ethnicity, and emerging adulthood: Retrospect and prospects. Emerging Adulthood, 1(2), 83-95.

Tanner, J. L. (2006). Recentering during emerging adulthood: A critical turning point in life span human development. In J. J. Arnett \& J. L. Tanner (Eds) Emerging adults in America: Coming of age in the $21^{\text {st }}$ century. Washington, DC: American Psychological Association.

Thelen, E., \& Smith, L. B. (1994). A dynamic systems approach to the development of perception and action. Cambridge, MA: Bradford Books/MIT Press

Twenge, J. M. (2013). The evidence for generation me and against generation we. Emerging Adulthood, 1(1), 11-16.

Van Lange, P. A., Kruglanski, A. W., \& Higgins, E. T. (Eds.). (2012).Handbook of Theories of Social Psychology: Volume Two (Vol. 2). Sage Publications.

Vygotsky, L. S. (1978). Mind in society: The development of higher mental process. Cambridge, MA: Harvard University Press. 
Waters, M. C., Carr, P. J., \& Kefalas, M. J. (2011). Introduction. In M. C. Waters, P. J. Carr, M. J. Kefalas, \& J. Holdaway (Eds.), Coming of age in America: The transition to adulthood in the twenty-first century. Berkeley: University of California Press.

Waters, M. C., Carr, P. J., Kefalas, M. J., \& Holdaway, J. (Eds.) (2011) Coming of age in America: The transition to adulthood in the twenty-first century. Berkeley: University of California Press. 